\title{
Modélisations des écoulements diphasiques dans les calculs d'accident de refroidissement des réacteurs nucléaires à eau sous pression Évolution et problèmes posés dans les applications pratiques
}

\author{
Mathematical models of two-phase flow for computing \\ loss of coolant accidents in pressurised water reactors. \\ Development and problems encountered in practice
}

\author{
R. Pochard et M. Réocreux \\ Institut de protection et de sûreté nucléaire, \\ Département d'études et de recherches en sécurité, \\ CEN - Fontenay-aux-Roses
}

\begin{abstract}
La prévision des accidents de refroidissement des réacteurs à eau sous pression met pratiquement toujours en jeu l'utilisation de modèles d'écoulements diphasiques. Pour évaluer la capacité de ces modèles à prévoir de tels accidents, il est nécessaire de bien cerner la nature même de ces modèles et la nature des problèmes posés dans leur application. Pour cela, on rappellera d'abord la méthodologie d'élaboration des modèles en mettant l'accent sur les hypothèses qui sont à leur base. Ceci permettra d'en fournir une classification pour les différents types de codes d'accident et de déterminer les actions nécessaires de validation expérimentale. Cette validation pose pour les études réacteurs un problème de transposition dont on examinera les difficultés et les moyens mis en œuvre pour les résoudre. Les capacités des modèles actuels à réaliser cette transposition seront enfin discutées mettant ainsi en évidence la nécessité d'améliorations quand à la pertinence physique des modélisations.
\end{abstract}

1. Élaboration des modèles d'écoulements diphasiques en vue de leur application aux calculs d'accidents réacteurs

L'élaboration de modèles d'écoulements diphasiques relève d'un processus de modélisation physique (cf. [J.M. Delhaye, 1968], [J. Bouré, M. Réocreux, 1972]) impliquant un certain nombre d'hypothèses qu'il est important d'identifier pour en bien connaitre les limites et les problèmes posés dans l'application pratique aux calculs d'accident.

\subsection{Equations locales instantanées}

La base de départ est constituée des principes de conservation. Ces principes sont d'abord écrits sous forme inté- grale. Des transformations mathématiques sont appliquées ensuite à ces formes qui conduisent à des équations décrivant le comportement local et instantané du fluide diphasique. Au cours de cette opération on fait une première hypothèse sur la nature possible du fluide présent dans un élément de volume qui définit le degré de détail visé dans la description physique choisie. On fait également une hypothèse sur la non prise en compte des propriétés de l'interface, hypothèse qui n'est que très peu restrictive dans les cas pratiques qui nous préoccupent.

\subsection{Equations moyennées}

Pour obtenir des équations applicables pratiquement il est nécessaire d'effectuer sur les équations précédentes des opérations de moyenne.

\subsubsection{Moyennes dans le temps}

Le premier type de moyenne est une moyenne dans le temps ou une moyenne statistique. Cette moyenne a pour but de "lisser " le caractère très aléatoire des écoulements diphasiques en écrêtant le "bruit " des fluctuations des paramètres locaux. Cette opération fait apparaître des coefficients de corrélation de type turbulent. Dans tous les modèles utilisés dans les codes de sûreté, ces coefficients sont toujours pris égaux à 1 .

Cette hypothèse n'a évidemment de sens que si le "bruit " que l'on désire écrêter reste très inférieur au signal moyen, ce qui n'est pas le cas dans de nombreuses situations (par exemple annulation des débits, phénomène de condensation brutale à l'injection...). Il est souhaitable aussi que la constante de temps servant à cette moyenne soit faible par rapport à la rapidité du transitoire. On peut pour s'affranchir de ce problème utiliser des moyennes statistiques, mais il se pose alors un problème pratique d'interprétation des signaux mesurés dans des expériences par nature uniques. Un point très important et en général 
totalement oublié est que cette moyenne est appliquée également aux termes de transfert. Les lois physiques spécifiant ces termes vont donc inclure la notion de constante de temps ce qui peut rendre difficile sinon impossible une bonne prédiction de certains phénomènes aléatoires ou dépendants de stabilité d'états physiques.

\subsubsection{Schématisation géométrique}

A partir des équations moyennées temporellement, il est le plus souvent nécessaire, afin de simplifier le modèle, d'utiliser une schématisation géométrique. Nous distinguerons ici la schématisation " théorique " et la schématisation "pratique ".

La schématisation " théorique " consiste, suf les équations moyennées temporellement et constituant un modèle à 3 dimensions d'espace, à opérer des opérations mathématiques de moyennes géométriques. Les moyennes peuvent être effectuées sur des volumes, des sections droites ou des segments conduisant respectivement à des modèles à 0,1 ou 2 dimensions. Ces opérations sont parfaitement rigoureuse mais il apparaît dans ces modèles des coefficients de corrélation spatiaux qui sont en général pris égaux à 1 . Pour être valable, cette dernière hypothèse demande l'existence de profils suffisamment plats sur l'élément géométrique considéré. Pour les écoulements diphasiques cette hypothèse est une hypothèse "dure". En effet, outre les cas où comme en écoulement monophasique les profils sont connus pour être assez marqués, s'ajoutent en écoulement diphasique les cas où l'une des phases présente des distributions très non uniformes (la vapeur par exemple dans des situations avec chauffage, certaines configurations d'écoulement). Ces distributions peuvent alors perturber les profils de l'ensemble des paramètres dans des situations hydrauliques où en monophasique les profils seraient effectivement plats. Néanmoins, faute souvent de résultats expérimentaux, cette hypothèse est faite bien que constituant une approximation importante.

Le deuxième niveau de schématisation géométrique se situe au niveau de la représentation du circuit. L'application pratique des équations moyennées précédemment de façon théorique, doit faire appel à une méthode numérique qui va discrétiser les équations. La définition des mailles de cette discrétisation à partir de la configuration géométrique réelle constitue ce que nous appellerons la schématisation "pratique ". Cette opération dont l'importance est souvent oubliée, peut être délicate car elle demande une "stylisation avancée " des géométries réelles.

Comme pour les moyennes temporelles, il faut rester conscient que ces moyennes géométriques s'appliquent aussi aux termes de transfert dont les expressions devront inclure ces notions d'échelle et de " stylisation " géométrique.

\subsection{Hypothèses additionnelles}

Pour fermer le système on fait généralement un certain nombre d'hypothèses additionnelles :

- les termes liés à la viscosité interne aux deux phases sont négligés dans les bilans de quantité de mouvement et d'énergie. Cette hypothèse est en général justifiée;
- les termes de conduction interne aux deux phases sor négligés. Cette hypothèse peut poser un problème lors qu'on veut décrire des gradients thermiques important (front de condensation par exemple lors de l'interventio des injections de secours);

- les équations d'état du fluide valables en toute rigueu pour les paramètres locaux et instantanées sont appliquée aux paramètres moyennés. Les erreurs introduites par cett hypothèse sont du même type que celles faites au nivea des coefficients de corrélation spatiaux;

- jusqu'à un passé récent, les modèles ont fait l'hypc thèse d'égalité des pressions moyennes dans chacune de phases et à l'interface. Cette hypothèse est très forte a niveau des transferts de quantité de mouvement et a ét remise en cause conduisant soit au développement $d$ modèles théoriques nouveaux, soit à la prise en compt dans les modèles existants de certains effets d'inégalité de pressions en particulier lorsque l'écoulement est stratifit cas où ces différences de pression sont connues.

\subsection{Différents types de modèles}

L'ensemble des opérations précédentes conduit naturelle ment à décrire les écoulements diphasiques par un modèl à six équations (modèle à deux fluides) qui permet 1 calcul des paramètres caractéristiques de chacune de phases ainsi que le taux de présence de ces phases (pa exemple taux de vide, pression, vitesses et températures d gaz et du liquide). Ce modèle permet donc de décrire 1 déséquilibre mécanique (écart entre les vitesses phasiques et les déséquilibres thermiques (écarts entre la températur de saturation et les températures phasiques). Pour obteni l'évolution de ces paramètres il est nécessaire de spécifie par des lois les termes de transfert qui apparaissent dan le modèle à savoir les transferts de masse, de quantité d mouvement et d'énergie qui ont lieu entre les phases $\epsilon$ entre chacune des phases et la paroi.

D'autres modèles sont utilisés où des hypothèses sur le déséquilibres ont permis de supprimer une ou plusieur équations de conservation. C'est ainsi qu'ont été écrits de modèles à équilibre thermodynamique de l'une ou de deux phases, des modèles à écarts de vitesse phasiqu calculés algébriquement (drift - flux par exemple), de modèles homogènes où les vitesses phasiques sont suF posées égales. L'intérêt de ces modèles est d'utiliser u nombre réduit de lois de transfert d'où une complexit moindre. Néanmoins, cette réduction résulte en fa d'hypothèses tacites sur des formes spécifiques de lois $d$ transfert [Réocreux, 1974] dont on verra au paragraphe les conséquences au niveau de l'applicabilité aux calcul de sûreté.

\section{Evolution des modèles utilisé dans les codes d'acciden}

\subsection{Codes de première génération}

Ces codes dont les premières versions datent d'une dizain d'années sont toujours utilisés dans de nombreuses évaluє tions d'accident. Le code type de cette catégorie est le cod 
américain RELAP 4 [Katsma et al., 1978] qui a été utilisé dans tous les pays.

\subsubsection{Modèles physiques de RELAP4}

Modèle d'écoulement de base : La base de ce modèle est le modèle homogène équilibré. Les phases $\mathrm{y}$ sont donc supposées à l'équilibre mécanique (vitesses égales) et à l'équilibre thermodynamique (saturation). L'écoulement est alors complètement décrit par les trois équations de conservation (masse, quantité de mouvement, énergie) appliquées au mélange et complétées par un jeu de lois de transfert entre parois et mélange (frottement et échanges thermiques). Compte tenu des limitations de ce modèle notamment en cas de stratification à faible vitesse, des modèles de déséquilibre mécanique ont été introduits de façon algébrique dans les versions successives du code. Les modèles disponibles dans la dernière version (Mod. 6) sont des modèles de montée de bulles et un modèle de glissement. Les modèles de montée de bulles présupposent l'existence d'un niveau, calculent son évolution dans le volume considéré de la distribution du taux de vide dans la partie inférieure du volume. Le modèle de glissement calcule une différence de vitesses à la jonction des volumes et modifie en fonction de ces vitesses le titre du mélange qui entre dans le volume récepteur et qui intervient dans l'équation d'énergie. Ces deux types de modèles sont initiés sur option par l'utilisateur, ce qui demande à ce dernier de choisir la période du transitoire et la portion du circuit où l'une ou/et l'autre de ces options doivent être utilisées.

Modèles particuliers. Ces modèles sont rajoutés au modèle d'écoulement de base pour décrire des phénomènes particuliers, ce sont principalement :

- les modèles de débit critique qui calculent par différentes corrélations (HEM, MOODY, HENRY par exemple) le débit maximum à la brêche en fonction des conditions de stagnation amont

- le modèle pompe qui est un modèle point calculant hauteur et couple en fonction des caractéristiques simple phase et double phase pleinement dégradées.

Couplage du modèle d'écoulement: Pour évaluer les échanges thermiques le modèle d'écoulement est couplé à un calcul de conduction dans les parois. Ces parois peuvent être multicouches, avec ou sans génération de puissance, le maillage pour le calcul de conduction pouvant être choisi par l'utilisateur. Un calcul de neutronique permet d'évaluer, le cas échéant, la génération de puissance.

\subsubsection{Schématisation géométrique et numérique}

Les équations sont moyennées sur des volumes pour les bilans de masse et d'énergie. L'équation de quantité de mouvement est écrite à la jonction de ces volumes de base en opérant une moyenne sur un volume décalé allant d'un centre à l'autre des deux volumes de base adjacents. Cette schématisation revient pour les tuyauteries à une schématisation ID à maille décalée où la discrétisation spatiale présenterait une forme particulière.

\subsection{Codes de deuxième génération}

Ces codes ont été écrits pour pallier les simplifications trop importantes des codes de première génération. Ces codes sont en cours de développement ou d'amélioration. Les codes types de cette catégorie sont les codes américains TRAC [D. Liles, J. Mahaffy et al., 1981] (version disponible TRAC PD2, TRAC PF1 $\bmod 0$, en cours de développement TRAC PF1 mod 1), RELAP 5 [V. Ransom et al., 1982] (version disponible mod 1, en cours de développement mod 2), COBRATF (en cours de développement) et le code français CATHARE [J.M. Miraucourt, G. Houdayer, 1983] [J.C. Rousseau, 1983]. (La livraison de CATHARE version 1 est prévue pour début 1984).

\subsubsection{Modèles physiques}

Modèles d'écoulement de base. Ces modèles sont des modèles à déséquilibre thermique et mécanique. Les variantes qui ont été successivement utilisées sont :

- pour TRAC PD2 dans les composants autres que la cuve un modèle à 5 équations $(2 \mathrm{M}, 1 \mathrm{Mvt}, 2 \mathrm{E})$ décrivant les déséquilibres thermiques et associé à une relation de différence de vitesse (drift flux)

- pour RELAP $5 \bmod 1$ un modèle à 5 équations $(2 \mathrm{M}$, $2 \mathrm{Mvt}, 1 \mathrm{E})$ décrivant le déséquilibre mécanique et le déséquilibre thermique de l'une des phases, la phase la moins présente étant supposée à la saturation

- pour TRAC PD2 dans la cuve, TRAC PF1 et CATHARE le modèle complet à deux fluides $(2 \mathrm{M}, 2 \mathrm{Mvt}, 2 \mathrm{E})$ incorporant pour TRAC PF1 et CATHARE l'effet des différences de pression entre phases, en particulier en cas de stratification dans une tuyauterie horizontale.

On constate donc une convergence des modèles vers le modèle complet à deux fluides. Ce modèle comporte la description de tous les transferts, en particulier des transferts entre phases qui ne figuraient pas dans les modèles des codes de première génération. Pour les transferts entre paroi et fluide. qui figuraient dans ces derniers, leurs expressions dans les codes américains de deuxième génération n'ont pas sensiblement évolué par rapport à celles utilisées dans RELAP4, en particulier pour les échanges thermiques.

Une mention particulière doit être faite pour le code COBRA-TF qui utilise un modèle à deux fluides mais également à deux champs liquide c'est-á-dire où la phase liquide y est traitée sous deux formes séparées : film et gouttelettes.

\section{Modèles particuliers.}

- Les modèles d'écoulement des codes de deuxième génération contiennent de façon générique la description du débit critique qui peut être obtenu en calculant l'évolution de l'écoulement au voisinage de la brêche. Néanmoins, des considérations de temps de calcul (nécessité d'un maillage fin et coûteux) et les résultats moins bons qu'espérés, ont conduit à réaliser un modèle spécifique (RELAP5) qui a été introduit en option dans la dernière version de TRAC.

- Pour la pompe, le code TRAC a repris la modélisation du code de première génération RELAP4. Dans RELAP5 et CATHARE1 les grandeurs caractéristiques de la pompe sont introduites sous forme de termes sources dans les 
équations courantes de l'écoulement (modélisation restant de même niveau que celle de RELAP4).

- Des modules spécialisés pour les branchements (Tés) et pour traduire les conditions limites, ont dû être écrits afin d'assembler les différentes parties de circuit.

Couplage du modèle d'écoulement: Comme pour les codes de première génération, le modèle d'écoulement est couplé à des calculs de conduction dans les parois avec de façon générale une souplesse moindre dans la représentation de ces parois au moins pour les premières versions.

\subsubsection{Schématisation géométrique et numérique}

Après quelques tentatives non concluantes de méthodes numériques différentes, tous les codes utilisent maintenant une représentation à maille décalée et à cellule donneuse. Les équations de masse et d'énergie sont écrites en des points appelés scalaires; les équations de quantité de mouvement sont écrites en des points dits vectoriels et décalés d'une demi-maille. Cette représentation est appliquée :

- soit à une schématisation à 1 dimension (TRAC PD2 dans les boucles seulement, TRAC PF1 dans les boucles et dans la cuve sur option, RELAP5 dans l'ensemble du circuit, CATHARE dans les boucles, le cœur et le downcomer

- soit à une schématisation à 3 dimensions (dans la cuve pour TRAC PD2, TRAC PF1 sur option et COBRA) dégénérant en 2 dimensions pour le downcomer.

Parmi les schématisations des codes de deuxième génération qui sont en général $1 \mathrm{D}, 2 \mathrm{D}$ ou $3 \mathrm{D}$, il faut noter la représentation OD à bivolumes de CATHARE qui peut être utilisée pour les plénums inférieur, supérieur, le dôme etc. (voir [J.M. Miraucourt, 1983].

3. Problèmes physiques posés par le.calcul d'accidents réacteur

\subsection{Ajustement des modèles et transposition d'échelle}

La démarche d'élaboration des modèles d'écoulement diphasique rappelée au paragraphe 1 a fait apparaître :

- un certain nombre d'opérations mathématiques parfaitement rigoureuses;

- des hypothèses qui ont été recensées et dont on a fait une première discussion de la validité.

Ceci a conduit à des modèles où restaient à spécifier des lois de transfert (transferts de masse, frottements, échanges thermiques). En écoulement diphasique ces lois ne sont jamais accessibles par une seule démarche théorique. Il est alors nécessaire pour écrire et qualifier ces lois de transfert, de s'appuyer sur des expériences en les ajustant au moyen de coefficients plus ou moins empiriques pour retrouver les résultats expérimentaux.

Deux cas se présentent alors :

- l'expérience sur laquelle on ajuste ces lois est parfaitement représentative du phénomène que l'on veut décrire. Dans ce cas n'importe quel type d'ajustement permettant de retrouver les résultats expérimentaux est satisfaisant;
- l'expérience n'est pas totalement représentative des phénomènes à décrire. C'est ce cas qui est le plus fréquemment rencontré dans la description des accidents réacteur en particulier du fait qu'il n'est souvent pas possible de représenter expérimentalement les phénomènes à l'échelle 1. Dans l'utilisation des modèles pour le cas pratique réacteur, il est alors nécessaire d'effectuer une transposition d'échelle et de s'assurer du maintien de la validité des lois de transfert dans cette transposition. Ce problème serait résolu si l'on connaissait les lois de similitude mais cela est très rarement le cas en écoulement diphasique.

\subsection{Discussion des capacités des modèles à la trans- position d'échelle}

\subsubsection{Influence des hypothèses d'élaboration des modèles}

Comme il a été montré au paragraphe 1, les hypothèses qui ont été utilisées dans l'élaboration des modèles ne sont souvent que des approximations grossières mais nécessaires faute de résultats expérimentaux suffisamment détaillés (profils par exemple). Lorsqu'on ajuste les lois de transfert sur les expériences, on va corriger en quelque sorte par une compensation d'erreurs les défauts intrinsèques aux modèles introduits par ces hypothèses. Il est alors très difficile de savoir comment cette compensation va se conserver dans la transposition. Il y a de fortes chances d'ailleurs que cette compensation ne se conserve pas, d'où l'importance qu'il y aurait, dans certains cas cruciaux, à pouvoir disposer d'analyse physique et de résultats expérimentaux qui pourraient réduire ces hypothèses de base des modèles.

\subsubsection{Nature des lois de transfert}

Plusieurs techniques d'ajustement des lois de transfert sur les expériences sont utilisées :

- la première consiste en un ajustement totalement empirique du type méthode des moindres carrés par exemple :

- la deuxième se veut complètement mécanistique, c'est-à-dire décrivant de façon théorique et complète les phénomènes. Cette description bute en fait toujours sur la détermination de paramètres physiques qui ne peut être faite que de façon empirique;

- la troisième est une voie intermédiaire : il s'agit d'établir des corrélations par nature empirique, mais basées sur la compréhension mécanistique des phénomènes que l'on a.

Il est clair que dans la transposition, les lois établies de la première façon ne donnent absolument aucune garantie. La qualié de la transposition dépendra de la compréhension physique qui sera intégrée à la loi et qui permettra de prendre en compte le poids des différentes composantes physiques qui ne manqueront pas de changer dans la transposition; l'exemple le plus commun de changement dans la transposition étant les effets surfaciques qui, vis-à-vis des effets volumiques, ont un poids plus élevé à faible échelle qu'à grande échelle. Dans cette optique seules les deux dernières techniques d'ajustement peuvent satisfaire aux problèmes de transposition. 


\subsection{Stratégie de qualification et de vérification des modèles}

Pour tenter de résoudre ce problème de transposition d'échelle qui est un problème crucial pour l'évaluation des accidents réacteurs, on utilise la stratégie suivante :

- Les lois de transfert sont établies et ajustées à partir d'expériences, en général à faible échelle où l'on reproduit de façon aussi séparée que possible les phénomènes se produisant sur le réacteur. Cette action de qualification sur expérience dites analytiques ou à effet séparé, doit, pour être crédible, conduire à un jeu de lois de transfert qui soit général et cohérent pour l'ensemble des conditions expérimentales rencontrées;

- Les modèles ainsi établis sont vérifiés sur des expériences globales ou systèmes qui représentent à une échelle en général plus grande que celle des expériences analytiques, l'ensemble complet du réacteur. Cette vérification permet de s'assurer que les modèles sont capables de coupler les phénomènes et de faire une première transposition d'échelle. Cette vérification doit, pour être crédible, s'effectuer à modèles figés. La vérification ainsi faite constitue le dernier point de contrôle avant d'extrapoler à l'échelle du réacteur.

\subsection{Evaluation de l'applicabilité des modèles aux évaluations réacteurs}

\subsection{Modes d'utilisation des modèles pour les évalua- tions réacteurs}

Il est clair que les modèles du type modèle homogène utilisés dans les codes de première génération peuvent n'avoir dans certaines situations qu'une pertinence physique limitée du fait des simplifications qui les ont générés. En conséquence, ces codes qui, historiquement, ont été utilisés pour le dimensionnement des réacteurs et des systèmes de sauvegarde ont donné le jour à deux types de versions :

- une version physique qui cherche à faire le meilleur calcul possible,

- une version d'évaluation qui, comportant un certain nombre d'hypothèses conservatives tant au niveau des conditions du transitoire qu'au niveau des lois physiques, vise à une évaluation majorante des conséquences de l'accident.

\subsubsection{Applicabilité des modèles d'évaluation}

Il s'agit de s'assurer pour ces modèles du conservatisme des résultats à l'échelle du réacteur. Compte tenu d'effets non linéaires très fréquents dans les écoulements diphasiques, il se peut qu'un calcul conservatif à faible échelle du fait d'une hypothèse considérée alors comme pessimiste, ne le soit plus à l'échelle du réacteur. Il est donc nécessaire de faire des études de sensibilité au niveau réacteur. Toutefois la meilleure garantie de ce conservatisme en reste son évaluation par comparaison avec un calcul physique d'où l'intérêt de tels calculs et des développements de code de deuxième génération plus physiques.

\subsubsection{Applicabilité des modèles physiques}

Pour ces modèles et les calculs physiques d'accident correspondants, on applique la stratégie énoncée au $\S 3.3$ Néanmoins tout modèle physique, aussi élaboré soit-il, ne prévoit la réalité physique qu'avec un certain degré d'incertitude. Le problème qui se pose alors est celui de l'évaluation de la marge d'incertitude à l'échelle du réacteur. Cela soulève de nombreuses difficultés pratiques qui font que ces calculs n'ont jamais encore été appliqués de façon autonome à des problèmes de dimensionnement.

\section{Discussion des capacités actuelles des modèles retenus dans les codes d'accident}

Il ne s'agit pas ici de donner une vue exhaustive des capacités des modèles retenus dans les codes d'accident mais seulement les tendances permettant d'apprécier les acquis et les améliorations à apporter aux modélisations des écoulements diphasiques.

\subsection{Codes de première génération}

Ces codes bien que de conception ancienne donnent des résultats assez satisfaisants dans le domaine des accidents grosse brêche en particulier pendant la phase de dépressurisation. L'hypothèse de base d'équilibre thermodynamique faite dans leur modèle d'écoulement les pénalise pour la description des phases d'injection et de renoyage.

Pour les situations accidentelles petite brêche où les phénomènes de séparation de phase sont prédominants, les options de glissement ou de modèles de montée de bulles permettent de retrouver au moins qualitativement les résultats. Dans les situations où les écoulements sont relativement homogènes (pompe en marche) le modèle de base homogène donne également de bons résultats. En dehors de ces cas extrêmes, les résultats sont problématiques.

\subsection{Codes de deuxième génération}

Les modèles de ces codes possèdent les potentialités requises pour des calculs dans tout le spectre des accidents y compris les situations accidentelles rencontrées au cours des procédures. Néanmoins, les résultats obtenus ne sont pas toujours du niveau espéré bien qu'en amélioration constante. La raison essentielle en est le manque de qualification des lois de transfert rendue difficile par leur complexité et par le fait que leur degré de sophistication dépasse souvent les capacités actuelles des mesures (effets 2D ou 3D par exemple).

\section{Conclusions}

L'application pratique des modèles d'écoulements diphasiques dans les codes d'accident réacteur pose le problème 
crucial de la transposition d'échelle. Ce problème a conduit à mettre en place une stratégie de qualification et de vérification sur expériences analytiques et systèmes. Parallèlement à cette stratégie, il est nécessaire de bien cerner les aspects fondamentaux qui pourraient remettre en cause la transposition et pour cela il faut revenir aux hypothèses théoriques de base qui ont permis l'élaboration des modèles.

Il apparaît alors qu'une bonne transposition et donc une meilleure applicabilité aux études de sûreté ne peuvent être acquises que par une meilleure compréhension physique des phénomènes, condition même d'une meilleure quantification. Les codes de deuxième génération constituent en cela un progrès appréciable mais ce progrès reste actuellement relativement potentiel par manque de qualification. Ces potentialités justifient que les efforts de développement alliés à une réflexion de caractère fondamental soient poursuivis.

\section{Références bibliographiques}

J. BOURE, M. RÉOCREUX. - General Equations of TwoPhase flows, Application to critical flows and to non-steady flows. Fourth All-Union Heat Transfer Conference (Minsk 1972).

K. KATSMA et al. - RELAP $4 \bmod 6$ - a computer program for transient thermal hydraulic analysis of nuclear reactors and related systems. CDAPTR 03, Idaho-Falls (Jan 1978).

D. LILES, J. MAHAFFY et al. - TRAC PF1 - an advanced best estimate computer program for pressurized water reactor analysis. LANL (1981).

J.M. MIRAUCOURT. - Modélisation des volumes de CATHARE. Communication à la Société hydrotechnique de France (16, 17 nov. 1983) La Houille Blanche, $\mathrm{n}^{\circ}$ 3/4-1984.

J.M. MIRAUCOURT, G. HOUDAYER. - CATHARE : Description, exemple de calcul d'un accident complet de réacteur PWR. Communication à la Société Hydrotechnique de France (16, 17 nov. 1983) La Houille Blanche, $n^{\circ} 3 / 4-1984$

V. RANSOM et al. - RELAP 5 Mod 1 Code manual, Vol 1 System models and numerical methods, Vol 2 Users guide and input requirement, NUREG/CR-1826, EGG-2070 (1982).

M. REOCREUX. - Contribution à l'étude des débits critiques en écoulement diphasique eau-vapeur. Thèse de Doctorat-esSciences, Université Scientifique et Médicale de Grenoble (1974).

J.C. ROUSSEAU. - Le modèle de base de CATHARE : qualification physique et performance. Communication à la Société Hydrotechnique de France (16, 17 nov. 1983). La Houille Blanche, $n^{\circ} 3 / 4-1984$. 